\title{
Immune Function Testing
}

National Cancer Institute

\section{Source}

National Cancer Institute. Immune Function Testing. NCI Thesaurus. Code C116682.

The use of a variety of different tests, including blood count and chemistry, urinalysis, and imaging to establish the status of an individual's immune system function. 